\title{
Dynamically Adjusting Migration Rates for Multi-Population Genetic Algorithms
}

\author{
Tzung-Pei Hong, Wen-Yang Lin \\ Dept. of Computer Sci. and Info. Engineering \\ National University of Kaohsiung \\ Kaohsiung 811, Taiwan \\ tphong@nuk.edu.tw, wylin@nuk.edu.tw
}

\author{
Shu-Min Liu, Jian-Hong Lin \\ Dept. of Information Management \\ I-Shou University \\ Kaohsiung 840, Taiwan \\ Shumin.Liu@msa.hinet.net, jhlin@isu.edu.tw
}

\begin{abstract}
In this paper, the issue of adapting migration parameters for MGAs is investigated. We examine, in particular, the effect of adapting the migration rates on the performance and solution quality of MGAs. Thereby, we propose an adaptive scheme to adjust the appropriate migration rates for MGAs. If the individuals from a neighboring sub-population can greatly improve the solution quality of a current population, then the migration from the neighbor has a positive effect. In this case, the migration rate from the neighbor should be increased; otherwise, it should be decreased. According to the principle, an adaptive multi-population genetic algorithm which can adjust the migration rates is proposed. Experiments on the 0/1 knapsack problem are conducted to show the effectiveness of our approach. The results of our work have illustrated the effectiveness of self-adaptation for MGAs and paved the way for this unexplored area.
\end{abstract}

Keywords: Soft computing, genetic algorithm, multipopulation, parameter adaptation, migration rate.

\section{Introduction}

Genetic Algorithms (GAs) are robust search and optimization techniques that were developed based on the ideas and techniques from genetic and evolutionary theory. Historically, genetic algorithms are characterized as an evolutionary scheme performed on a single population of individuals.

Multi-population genetic algorithms (MGAs) [7] is an extension of traditional single-population genetic algorithms (SGAs) by dividing a population into several sub-populations within which the evolution proceeds and individuals are allowed to migrate from one sub-population to another. This model is analogous to that used in parallel island GAs [2], without emphasizing the parallelism issue. Indeed, the MGA is more identical to the natural genetic evolution. For example, the human species consist of groups distant from each other while individuals within a group would migrate to another group. A typical MGA can be described as Figure 1. First, each sub-population evolves independently till some specific generations to reach the theoretical equilibrium, resulting in many local optimal solutions in sub-populations. Each sub-population then migrates with its neighborhood(s). This punctuates the equilibrium and forces each sub-population evolving again to escape the local optimum and exploiting toward the global optimum.

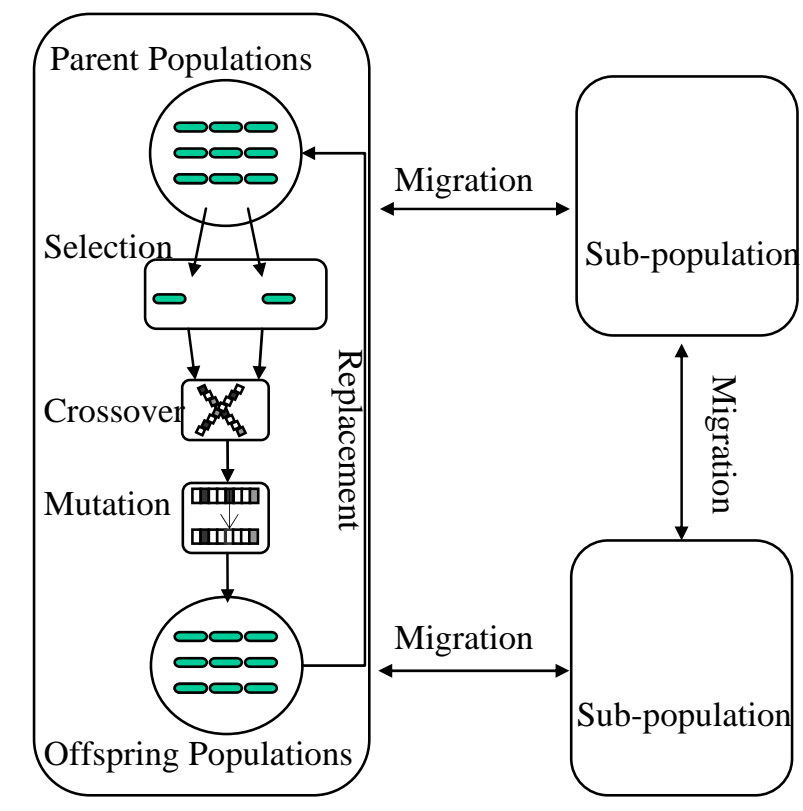

Figure 1: A typical MGA with three subpopulations

Until recently, researchers have found that MGAs are more effective both in algorithmic cost and solution quality [6][10][13][14], such as:

1. It would shorten the number of generations needed to find the optimal or near optimal solution.

2. It usually can find more than one optimal solution.

3. It is more resistance to premature convergence.

Despite of these advantages, the behavior and performance of MGAs are still heavily affected by a judicious choice of parameters, such as connection topology (the topology that defined the connection between the subpopulations), migration policy, 
migration interval (a parameter that controls how often the migration occurs), migration rate (a parameter that controls how many individuals migrate), and population size (the number of subpopulations), etc. To our knowledge, most of the previous work focused on providing guidelines to choose those parameters empirically or rationally [3][4][13], and no work was devoted to the effect of adapting these parameters.

In this paper, the issue of adapting migration parameters for MGAs is investigated. We in particular examine the effect of adapting migration rates on performance and solution quality of MGAs. Thereby, we propose an adaptive scheme to adjust appropriate migration rates for MGAs. Experiments on the wellknown 0/1 knapsack problem showed that our approach was superior to MGAs with static migration intervals and migration rates.

The remaining parts of this paper are organized as follows. Related previous works are reviewed in Section 2. The proposed migration-rate adjusting algorithm is described in Section 3. The 0/1 knapsack problem used to illustrate the proposed algorithm is also presented there. Experimental results for showing the performance of the proposed algorithm are provided in Section 4. Conclusion and future works are given in Section 5.

\section{Previous Works}

Grosso [8] was probably the first one that gives a systematic study of multi-population genetic algorithms. The MGA model he considered consisted of five subpopulations and each exchanged individuals with all the others with a fixed migration rate. With controlled experiments, he observed that the migration rate had great effect on the performance of MGAs. MGAs with migration rate below a critical point would have a similar effect to that with no migration at all.

Another study conducted by Pettey et al. [15] used a similar model but with different migration strategy. The migration occurs after each generational evolution, for which a copy of the best individual in each subpopulation is sent to all its neighbors.

In a parallel implementation of MGAs on a 4-D hypercube proposed by Tanese [17], migration occurred at fixed intervals between processors along one dimension of the hypercube. The migrants were chosen probabilistically from the best individuals in each subpopulation, and replaced the worst individuals in the receiving subpopulations. From experiments of different migration intervals, she observed that migrating too frequently or too infrequently degraded the performance of the algorithm. Another work of Tanese [18] showed that MGAs with migration performed significantly better than those without migration. Recently, a study conducted by Belding [1] using Royal Road function exhibited a similar conclusion.

In experiments with the parallel MGA, Cohoon et al. [6] noticed that there was relatively little change between migrations, but new solutions were found shortly after individuals were exchanged.

In the majority of multi-population GAs, migration is synchronous, which means that it occurs at predetermined constant intervals. Grosso [8] was also the first one to consider an asynchronous migration scheme, where migration is enabled until the population was close to converge.

Braun [2] used the same idea and presented an algorithm where migration occurred after the populations converged completely. The same migration strategy was used later by Munetomo et al. [14], and so by Cant'u-Paz and Goldberg [5].

The traditional multi-population genetic algorithm fixes both the migration intervals and migration rates. It is stated as follows.

\section{The Multi-population Genetic Algorithm with Fixed Migration Intervals and Migration Rates:}

Initialize the parameters;

Generate $N$ sub-population $P_{1}, P_{2}, \ldots, P_{N}$ randomly;

generation $\leftarrow 1$;

while generation $\leqq$ max_gen do

for each sub-population $P_{i}$ do

Use a fitness function to evaluate each individual in $P_{i}$; Perform crossover in $P_{i}$;

Perform mutation in $P_{i}$;

Perform replacement in $P_{i}$;

endfor

generation $\leftarrow$ generation +1 ;

if (generation \% migration-interval $==0$ ) endwhile

Perform migration at the fixed migration rate;

\section{The Proposed Migration-Rate Adjusting Approach}

Traditional multi-population genetic algorithms use only a single migration interval and a migration rate to exchange individuals among sub-populations and to prevent premature. Different migration intervals and migration rates can, however, produce different fitness values, thus affecting the performance of the applied multi-population genetic algorithm. Designing a new multi-population genetic algorithm to automatically apply appropriate migration intervals and migration rates is then necessary.

The rationale behind the proposed algorithm is that if the individuals from a neighboring subpopulation can greatly improve the solution quality of a current population, then the migration from the neighbor has a positive effect. In this case, the migration rate from the neighbor should be increased; otherwise, it should be decreased. According to the principle, an adaptive multi-population genetic 
algorithm which can adjust the migration rates is proposed as follows.

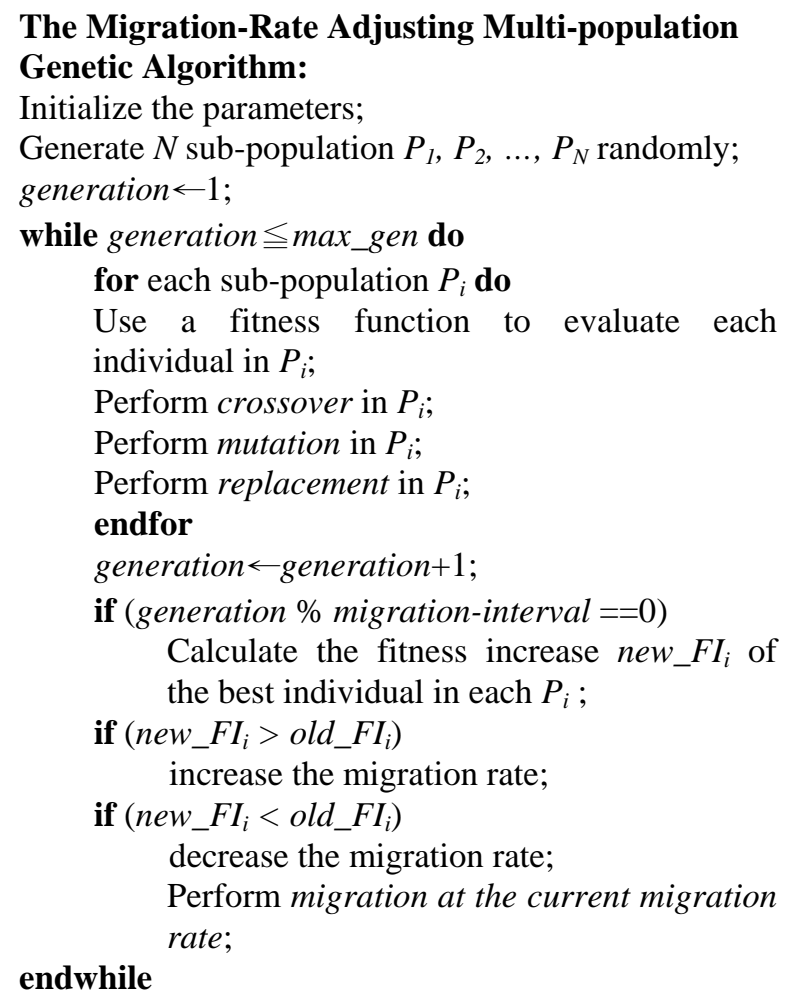

Note that in the above algorithm, the variable old_FI represents the fitness increase in the last migration interval. It is the difference of two fitness values in two neighboring migration intervals. Also different rules can be used to change the migration rate. The change may depend on the proportion of the fitness difference between two migration intervals or on a constant ratio.

The $0 / 1$ knapsack problem is used in this paper to illustrate how the proposed algorithm can overcome the traditional one. It belongs to the class of knapsacktype problems and is well known to be NP-hard [11]. The problem is stated as follows. Given a set of objects, $a_{i}$, for $1 \leq i \leq n$, together with their profits $P_{i}$, weights $W_{i}$, and a capacity $C$, the $0 / 1$ knapsack problem will try to find a binary vector $x=x_{1}, x_{2}, \ldots$, $x_{n}$, such that:

$$
\sum_{i=1}^{n} x_{i} \cdot W_{i} \leq C, \text { and } \sum_{i=1}^{n} x_{i} \cdot P_{i} \text { is maximal. }
$$

Following the suggestion made in [11], the data were generated with the following parameter settings: $v=10, n=250, W_{i}$ and $P_{i}$ are random(1..v), and $C=$ $2 v$, for which the optimal solution contained very few items. Here, $v$ is the maximum possible weight or profit.

To facilitate the study, we consider the most adopted model of MGAs as shown in Figure 1 [2][9]. There are $\gamma$ subpopulations, all of which are connected with as a ring structure. Individuals in MGA are migrated after every migrate interval of generations. Besides, the best-worst migration policy is used [1][8][11]. That is, the best $\rho$ (migration rate) of individuals in one subpopulation are selected to migrate to its neighbor subpopulations, and replace the worst individuals.

To be consistent with the crossover and mutation operators considered, the binary encoding scheme is used. Each bit represents the inclusion or exclusion of an object. It is, however, possible to generate infeasible solutions with this representation. That is, the total weights of the selected objects may exceed the knapsack capacity. In the literature, two different ways of handling this constraint violation [12] have been proposed. One way is to use a penalty function to penalize the fitness value of the infeasible candidate to diminish its chance of survival. Another approach is to use a repair mechanism to correct the representation of the infeasible candidate. In [12], the repair method was more effective than the penalty approach. Hence, the repair approach is adopted in our implementation.

The repair scheme adopted here is a greedy approach. All the objects in a knapsack represented by an overfilled bit string are sorted in decreasing order of their profit-weight ratios. The last object is then selected for elimination (the corresponding bit of " 1 " was changed to " 0 ”). This procedure is executed until the total weight of the remaining objects is less than the total capacity.

\section{Experimental Results}

Experiments on the above 0/1 knapsack problem are made for showing the performance of the proposed algorithm for adjusting migration rates based on fitness values. The parameters used in the proposed approach are set as follows.

- Number of populations: 16;

- Sub-population size: 30;

- Initial population: generated by random;

- Connection topology: ring;

- Crossover rate: 0.65;

- Mutation rate: 0.05;

- Selection method: tournament selection;

- Replacement method: keeping better individuals of old and new populations;

- Migration policy: selecting the best individuals to replace the worst ones of the neighbor subpopulations;

- Termination condition: 2000 generations;

- Number of experiments: 15 runs (get the average).

The migration rates are dynamically adjusted according to the improvement degrees from the neighbors. The initial migration rate is set at 0.4. Experimental results with dynamic migration and 
without migration are first compared. The fitness values are measured along different generations for migrations intervals fixed respectively at 1, 10, 20, 40 and 80 . The experimental results for the best fitness values are shown in Figure 2.

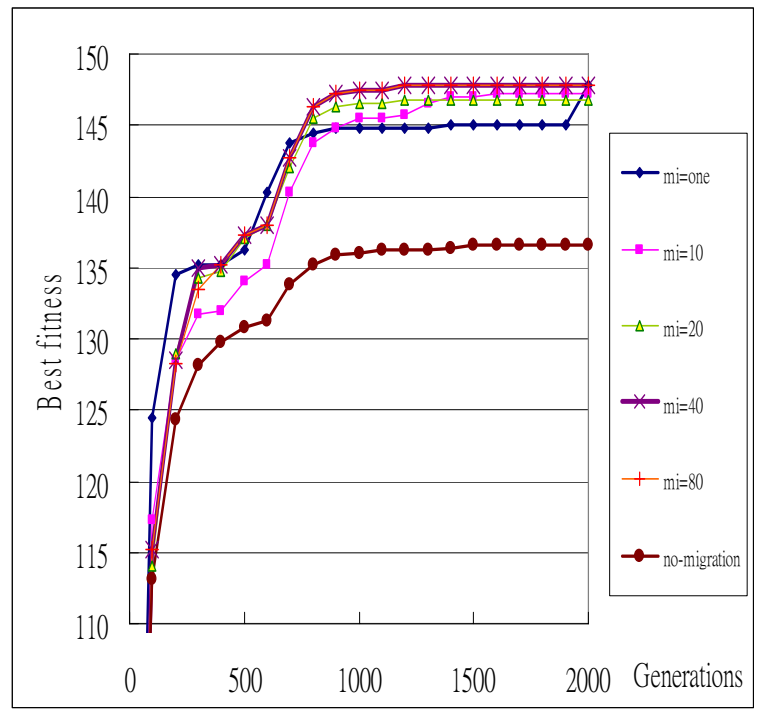

Figure 2: The best fitness values along with different generations for the above algorithm

It can be easily seen form Figure 2 that the experimental results with dynamic-rate migration are better than those without migration no matter what migration intervals are set at. The average fitness values along with different generations are shown in Figure 3.

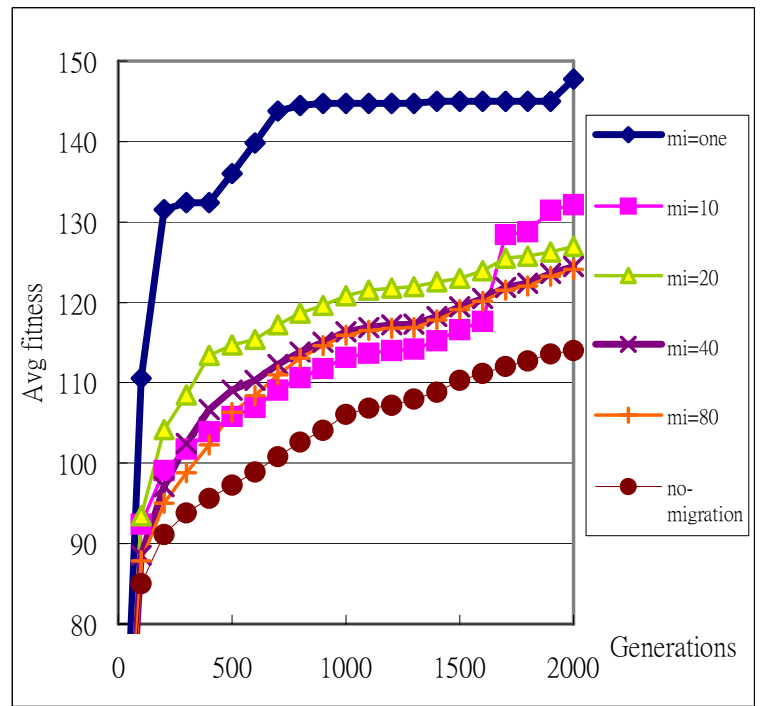

Figure 3: The average fitness values along with different generations for the above algorithm

It can be seen that the results with dynamic-rate migration are also better than those without migration. Besides, when the migration interval is fixed at one ( $m i=$ one), the algorithm generates the best effect.
Next, experiments are made for comparing the performance with and without dynamic migration-rate adjusting. When the migration interval is fixed at one, the results for different fixed migration rates $(0.1,0.2$, $0.4,0.8$, and one individual) and for dynamic migration rates are shown in Figure 4.

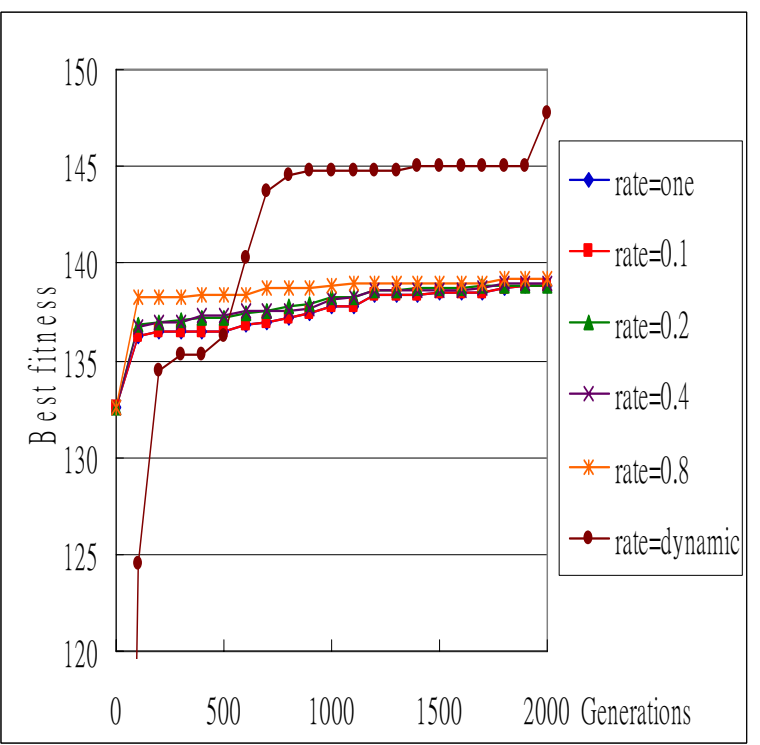

Figure 4: The best fitness values for different fixed migration rates and for dynamic migration rates when the migration interval is fixed at one

It can be easily seen from Figure 4 that the approach with dynamic migration-rate adjusting gets a better fitness value than those at fixed migration rates. The same experiments are then made for different intervals. When the intervals are set at 10, 20, 40 and 80, the results are respectively shown in Figures 5 to 8.

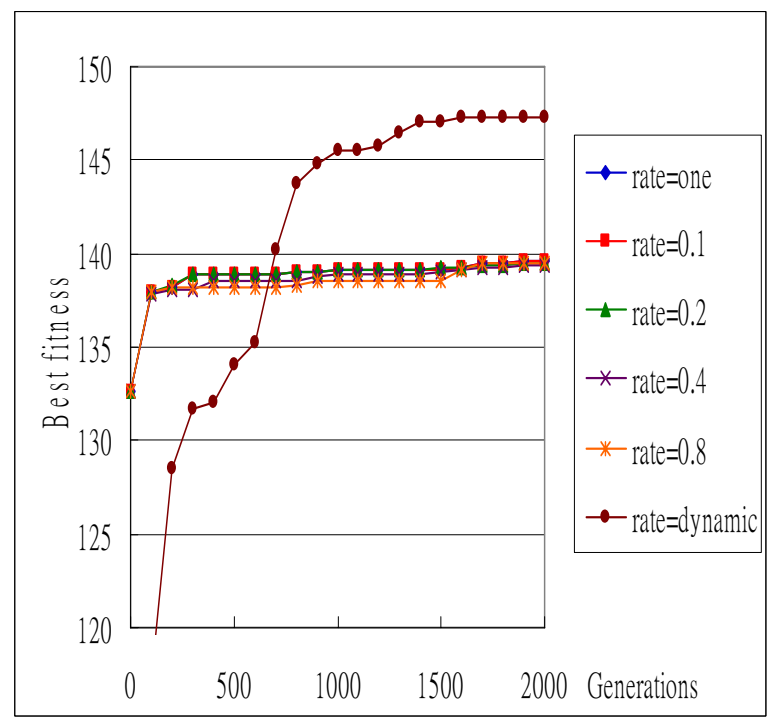

Figure 5: The best fitness values for different fixed migration rates and for dynamic migration rates when the migration interval is fixed at $\mathbf{1 0}$ 


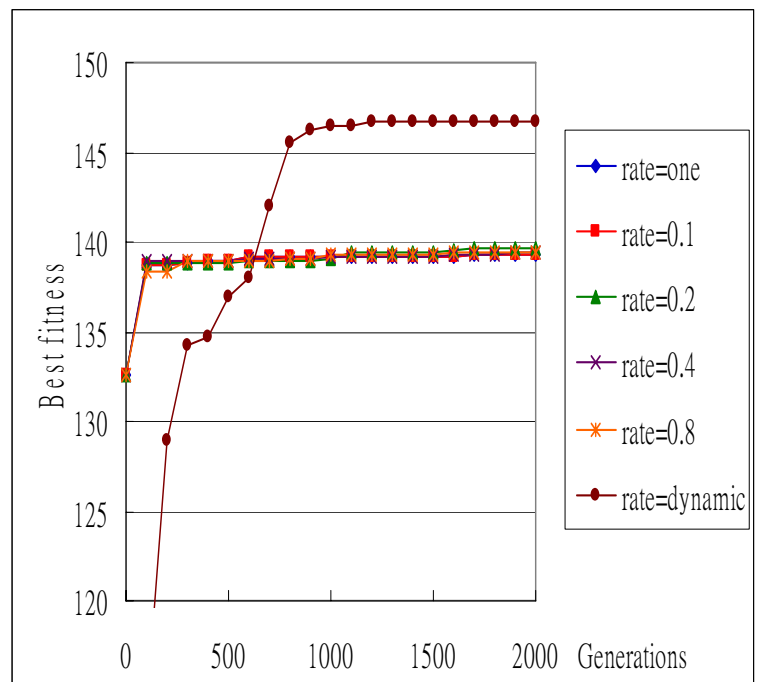

Figure 6: The best fitness values for different fixed migration rates and for dynamic migration rates when the migration interval is at 20.

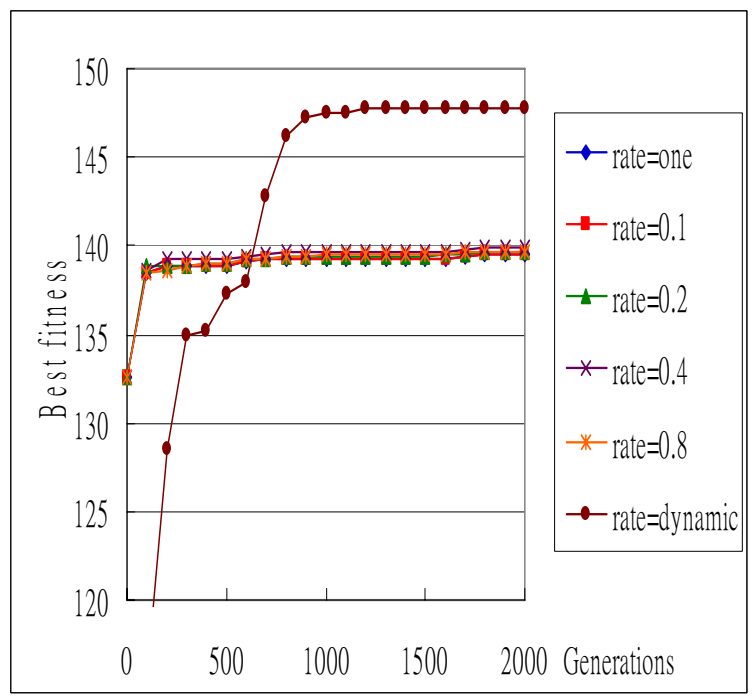

Figure 7: The best fitness values for different fixed migration rates and for dynamic migration rates

when the migration interval is fixed at $\mathbf{4 0}$

It can be concluded from these figures that the proposed algorithm has a good effect for all the different interval settings at 1, 10, 20, 40 and 80 . The experimental results for the average fitness values are similar to the above ones.

\section{Conclusions}

In this paper, we have studied the issue of adapting migration rates for MGAs, to improve performance and solution quality. An adaptive scheme has been devised, which adjusts migration rates. A preliminary study on the $0 / 1$ knapsack problem has showed that the proposed adaptive approach can compete with a static approach with the best-tuned migration rate and migration interval.

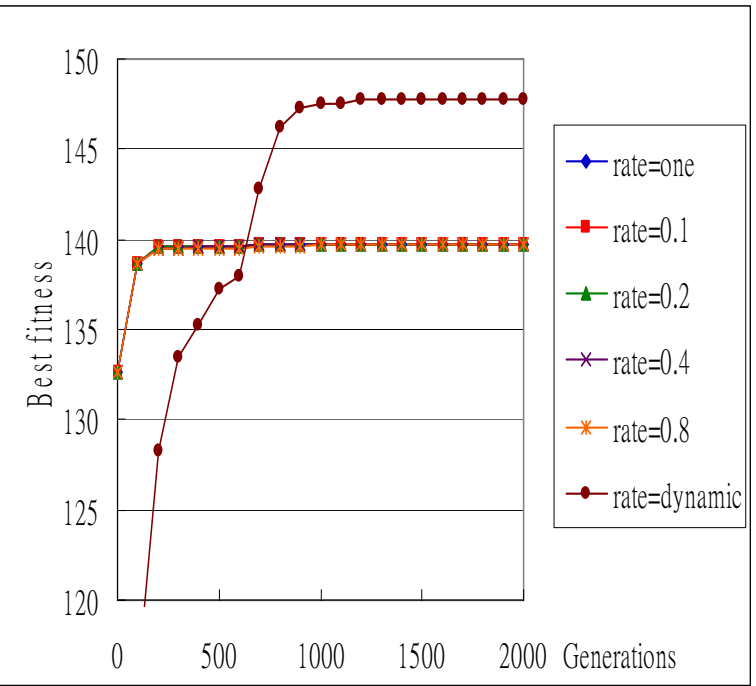

Figure 8: The best fitness values for different fixed migration rates and for dynamic migration rates when the migration interval is fixed at 80

As future works, we will conduct more experiments on other benchmarks, and devise methods to pursue an appropriate convergence threshold. We also will consider incorporating other representative parameters of MGAs, such as connection topology and migration policy, into our adaptive model.

\section{Acknowledgements}

This research was supported by the National Science Council of the Republic of China under contract NSC94-2213-E-390-005.

\section{References}

[1] T.C. Belding, "The distributed genetic algorithm revisited," in Proceedings of the Sixth International Conference on Genetic Algorithms, pp. 114-121, 1995.

[2] H.C. Braun, "On solving travelling salesman problems by genetic algorithms," in Proceedings of the First Workshop on Parallel Problem Solving from Nature, pp. 129-133, 1991.

[3] E. Cant'u-Paz, "A survey of parallel genetic algorithms," Calculateurs Paralleles, Reseaux et Systems Repartis, Vol. 10, No. 2, pp. 141-171, 1998.

[4] E. Cant'u-Paz, "Migration policies and takeover time in parallel genetic algorithms," in Proceedings of Genetic and Evolutionary Computation Conference, 1999.

[5] E. Cant'u-Paz and D.E. Goldberg, "Efficient parallel genetic algorithms: Theory and practice," Computer Methods in Applied Mechanics and Engineering. 
[6] J.P. Cohoon, S.U. Hegde, W.N. Martin, and D.S. Richard, "Punctuated equilibria: A parallel genetic algorithm," in Proceedings of the Second International Conference on Genetic Algorithms, pp. 148-154, 1987.

[7] J.P. Cohoon, W.N. Martin, and D.S. Richards, "Punctuated equilibria: A parallel genetic algorithm," in Proceedings of the Second International Conference on Genetic Algorithms, pp. 148-154, 1987.

[8] J.J. Grefenstette, "Parallel adaptive algorithms for function optimization,” Technical Report No. CS-81-19, Vanderbilt University, Computer Science Department, Nashville, TN, 1981.

[9] P.B. Grosso, Computer Simulations of Genetic Adaptation: Parallel Subcomponent Interaction in a Multilocus Model, Doctoral Dissertation, The University of Michigan, 1985.

[10] S.C. Lin, W.F. Punch III, and E.D. Goodman, "Coarse-grain parallel genetic algorithms: categorization and new approach,” in Proceedings of the Fifth International Conference on Genetic Algorithms, pp. 28-37, 1994.

[11] S. Martello and P. Toth, Knapsack Problems, Jonh Wiley, UK, 1990.

[12] Z. Michalewicz, Genetic Algorithms + Data Structures = Evolution Programs, SpringerVerlag, 1994.

[13] H. Muhlenbein, M. Schomisch, and J. Born, "The parallel genetic algorithm as function optimizer," in Proceedings of the Fourth International Conference on Genetic Algorithms, 1991.

[14] M. Munetomo, Y. Takai, and Y. Sato, "An efficient migration scheme for subpopulationbased asynchronously parallel genetic algorithms," in Proceedings of the Fifth International Conference on Genetic Algorithms, 1993.

[15] C.B. Pettey, M.R. Leuze, and J.J. Grefenstette, "A parallel genetic algorithm," in Proceedings of the Second International Conference on Genetic Algorithms, pp. 155-161, 1987.

[16] D. Schlierkamp-Voosen and H. Muhlenbein, "Adaptation of population sizes by competing subpopulations," in Proceedings of IEEE International Conference on Evolutionary Computation, pp. 330-335, 1996.

[17] R. Tanese, "Parallel genetic algorithm for a hypercube," in Proceedings of the Second International Conference on Genetic Algorithms, pp. 434-439, 1987.

[18] R. Tanese, "Distributed genetic algorithms," in Proceedings of the Third International Conference on Genetic Algorithms, pp. 177-183, 1989. 